\title{
Parameter Tuning Associated with Nonlinear Dynamics Techniques for the Detection of Cardiac Murmurs by Using Genetic Algorithms
}

\author{
E Delgado, J Jaramillo, AF Quiceno, G Castellanos \\ Control and Digital Signal Processing Group, National University of Colombia
}

\begin{abstract}
In this study, nonlinear dynamics techniques toward detecting cardiac murmurs from phonocardiograms (PCG) are used. With this purpose, a methodology for tuning parameters (reconstruction delay $-\tau$ and embedding dimension $-m$ ) involved in the reconstruction of a meaningful state space from scalar time series is presented, using genetic algorithms (GA), as well as constructing a metaalgorithm combined with support vector regression to adjust the GA parameters in order to decrease the computational cost. The forecasting capacity is used as cost function of the GA. The PCG records belong to the National University of Colombia, 360 beats were chosen by specialist, 180 normal and 180 with cardiac murmur evidence. The obtained results show that by using the tuned GA an efficient procedure for the consistent determination of $\tau$ and $m$ is achieved. Murmur detection by using nonlinear features was obtained with classification accuracy of $96 \%$ using a $k$ nearest neighbor classifier in cross-validation with 10 folds.
\end{abstract}

\section{Introduction}

Due to the complexity involved in cardiac dynamics [1], nonlinear dynamics techniques for detecting cardiac murmurs from phonocardiographic signals (PCG) have been proposed [2]. A topological equivalent corresponding to the true state space of a system can be reconstructed with the method of delays [3]. The embedding dimension $m$ and the reconstruction delay $\tau$ are mandatory information for the state space reconstruction, or embedding, of a series [4, 5]. Estimating these values allows for the construction of state vectors, also called regressors, which contain all the information about system dynamics [6]. However, in time series analysis there exist several difficulties. Usually, the attractor dimension is not known and therefore there is no idea about the minimal embedding dimension $m$. In another way, the reconstruction delay $\tau$ is not subject to the embedding theorems; in principle, arbitrary $\tau$ yields an embedding, and according to this, the main difficulty is related to the observability of interesting structures [7].
Several methods have been developed for estimating the embedding dimension: correlation dimension [8], false neighbors [9], Box-Couting [5], minimum dimension [10], etc. Concerning with the selection of the time delay, common approaches are: the use of the series autocorrelation, or the mutual information (MI) [11]. The goal is to select the regressor components, as uncorrelated or independent as possible. Genetic Algorithms (GA) have been widely used for tuning parameters taking into account an adequate cost function, for example, in [12], an approach based on evolutionary algorithms was developed to find parameters for optimal embedding and, the application of chaotic time series forecasting as cost function to build a nonlinear model from the dataset is proposed in [13]. However, when the task implies building the state space from a lot of different systems, computational complexity considerably increases.

In this study, the use of nonlinear dynamics techniques for characterization of FCG signals in the detection of cardiac murmurs is proposed. The problem lies in the adjustment of $\tau$ and $m$ of the nonlinear model, so we propose the use of GA to accomplish that task. The evaluation function is based on measuring the forecasting capabilities obtained with each pair of values for $m$ and $\tau$. A probabilistic approach is used for comparing the predicted and original attractors. In order to minimize the computational cost in the tuning procedure, the control parameters of the GA must be carefully selected as well. A methodology is presented where a meta-algorithm combined with support vector regression is used for tuning the GA control parameters.

\section{Materials and methods}

\subsection{Database}

The database is made up of 148 de-identified adult subjects, who gave their formal consent, and underwent a medical examination (the valve lesion severity was evaluated by cardiologists according to clinical routine). 50 patients were labelled as normal, while 98 with evidence of cardiac murmurs, caused by valve disorders (aortic stenosis, mitral regurgitation, etc). Besides, 8 recordings cor- 
responding to the four traditional focuses of auscultation (mitral, tricuspid, aortic and pulmonary areas) were taken for each patient in the phase of post-expiratory and postinspiratory apnea. Each record lasts $12 s$ and was obtained with the patient standing in dorsal decubitus position. Finally, 360 representative beats were chosen by specialist, 180 normal and 180 with evidence of cardiac murmur. An electronic stethoscope (WelchAllyn ${ }^{\circledR}$ Meditron model) was used to acquire the heart sounds simultaneously with a standard 3-lead ECG (the DII derivation was used because the QRS complex is clearly defined). Both signals were digitized at $44.1 \mathrm{kHz}$ with 16-bits per sample.

\subsection{Embedding theorem}

The state space reconstruction is based on the TimeDelay Embedding Theorem presented in [4], which can be written as follows [5]: Given a dynamical system with a $m$-dimensional solution space and an evolving solution $h(t)$, let $x$ be some observation $x(h(t))$. Let us also define the lag vector (with dimension $m$ and common time lag $\tau) \boldsymbol{x}(t) \equiv\left(x_{t}, x_{t-\tau}, x_{t-2 \tau}, \ldots, x_{t-(m-1) \tau}\right)$. Then, under very general conditions, the space of vectors $\boldsymbol{x}(t)$ generated by the dynamics contains all of the information of the space of solution vectors $\boldsymbol{h}(t)$. The mapping between them is smooth and invertible. This property is referred to as diffeomorphism and this kind of mapping is referred to as an embedding. Thus, the study of the time series $\boldsymbol{x}(t)$ is also the study of the solutions of the underlying dynamical system $\boldsymbol{h}(t)$ via a particular coordinate system given by the observable $x$.

The embedding theorem establishes that, when there is only a single sampled quantity from a dynamical system, it is possible to reconstruct a state space that is equivalent to the original (but unknown) state space composed of all the dynamical variables [14].

\subsection{Predicting chaotic time series}

If the data are a single time series, the first step is to embed them in a state space. If the attractor is of dimension $D$, a minimal requirement is that $m \geq D$. The next step is to assume a functional relationship between the current state $\boldsymbol{x}(t)$ and the future state $\boldsymbol{x}(t+T)=f_{T}(\boldsymbol{x}(t))$. To predict $x(t+T)$, a metric on the state space denoted by || || should be imposed, and find the $k$ nearest neighbors of $\boldsymbol{x}(t)$, i.e., the $k$ states $\boldsymbol{x}\left(t^{\prime}\right)$ with $t^{\prime}<t$ that minimize $\left\|\boldsymbol{x}(t)-\boldsymbol{x}\left(t^{\prime}\right)\right\|$. A local predictor is constructed, regarding each neighbor $\boldsymbol{x}\left(t^{\prime}\right)$ as a point in the domain and $x\left(t^{\prime}+T\right)$ as the corresponding point in the range. For convenience, the range is treated as a scalar, mapping $m$-dimensional states into one-dimensional values, although for some purposes it is desirable to let the range be $m$-dimensional. The fit can be made in any of several ways; for the work re- ported here, two approaches are used: when the regression problem is well-posed, least squares by singular value decomposition is used; on the other hand, when the $k$ nearest neighbors produce an ill-posed problem (the condition number of the matrix with the $k$ nearest neighbors is too large), the Tikhonov regularization method is used for estimating the regression value [15].

\subsection{Genetic meta-algorithm}

Genetic algorithms are computational methods inspired on natural selection and evolution precesses, frequently used for optimization tasks. However, for a maximum efficiency, it is necessary to adjust the control parameters (i.e., population size $r$, crossover rate $\chi$ and mutation rate 4 ) [16]. For this purpose, a tuning method called metaalgorithm (meta-GA) has shown to be an effective way to select the best values of control parameters [17, 18]; this method is composed by a low level GA, that is, the one that works on the specific optimization problem, and a high level $G A$, which is optimizing the control parameters of the first one, by using some evaluation function that reflects its performance in terms of accuracy and computational cost. The main difficulty involved with the meta-GA implementation is its own computational cost. For that reason, different methodologies has been proposed [19].

\subsection{Proposed procedure}

The PCG record is taken as a time series $x(t)$ and the evaluation function must be established in order to have a performance measure for each set of $m$ and $\tau$. This one will be given by the forecasting skills obtained by the reconstructed attractor of $x(t)$. Figure 1 depicts the proposed procedure for evaluating the reconstruction quality. $m$ and $\tau$ values for reconstructing the state space are found by using Algorithm 1 .

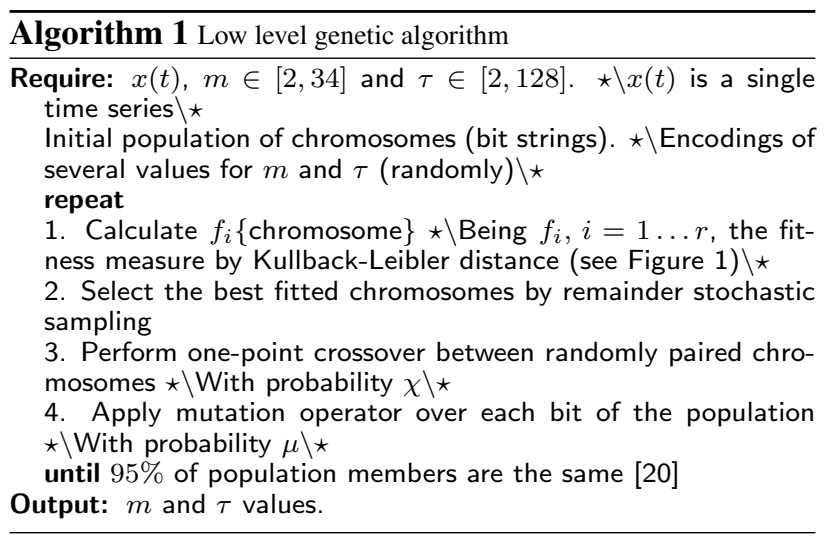

The proposed methodology for tuning the GA control parameters (see Figure 2) begins with the execution of 


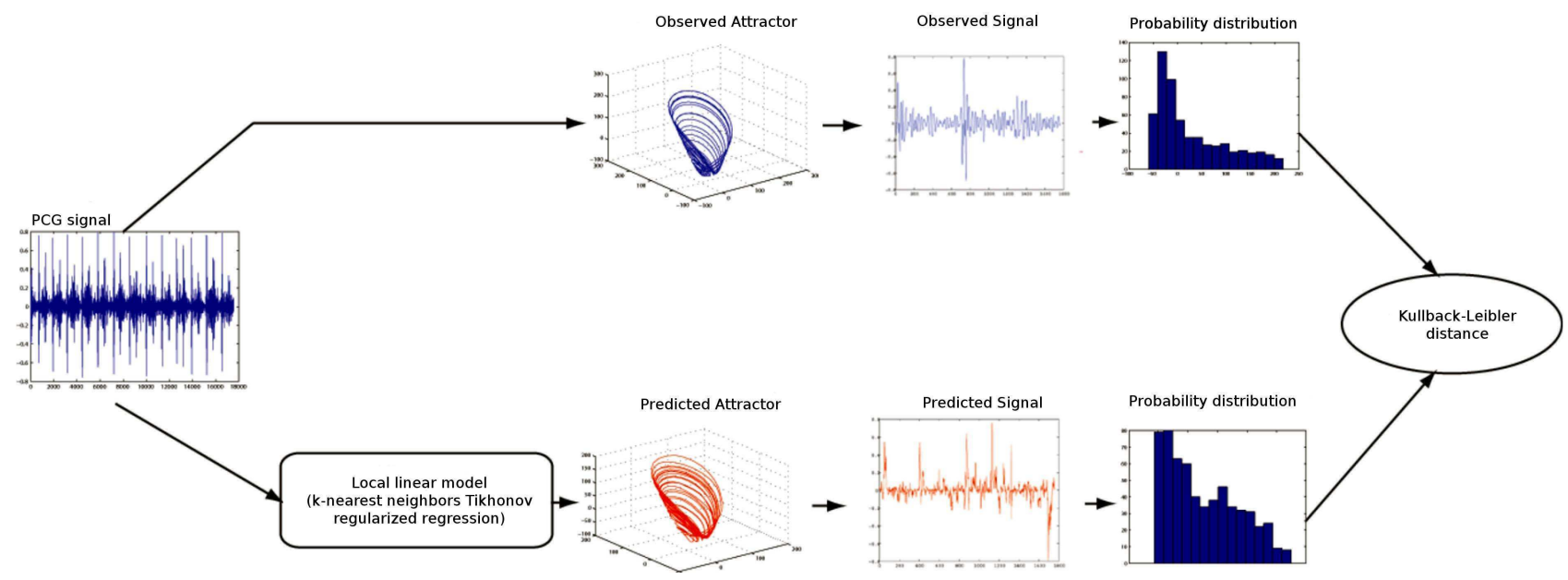

Figure 1. Evaluation function

Algorithm 1 (low level GA), with different sets of control parameters. For each execution, the evaluation of the found solution and the the total number of evaluations are recorded for constructing two regression surfaces (i.e., using the support vector regression method [21]). Thus, without executing the low level GA, the evaluation value and the number of evaluations can be estimated by using these surfaces. In this way, the high level GA can be optimized with the obtained surface, since it minimizes the number of evaluations and maximizes the evaluation value in lower processing time. For further details on this methodology, in [22] there is a wide explanation.

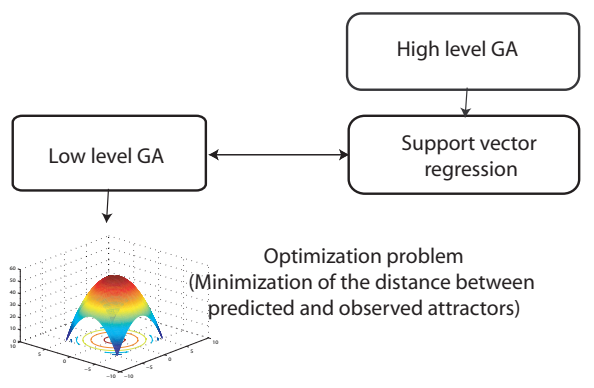

Figure 2. Control parameters optimization methodology

After attractor reconstruction, in order to detect cardiac murmurs, the following features are estimated over the whole training database:

Correlation Dimension $\left(D_{2}\right)$ : Calculation of $D_{2}$ is achieved by using the method proposed in [23], requiring a previous estimation of the correlation sum, $C(r)$. The function $\ln C(r)$ vs. $\ln (r)$ is evaluated for every PCG beat, estimating its scaling region by the derivation, $\operatorname{dln} C(r) / d \ln (r)$. Largest Lyapunov Exponent $\left(\lambda_{1}\right)$ : It gives a measure of chaos in the system, estimating the exponential separation of the trajectories. $\lambda_{1}$ can be estimated as the average rate of separation from the nearest neighbors like is proposed in [23].

Attractor probabilistic weighted variance $\left(\nu_{p w}\right)$ : It gives a measure of the attractor dispersion, weighted with the correspondent density from a state space box arrangement.

The classification strategy used for detecting cardiac murmurs from nonlinear features was $k$-NN, trained by using 10-fold cross-validation.

\section{Results}

Previous studies present excellent classification results well above $89 \% \pm 3.1$ when the attractors are reconstructed using the same embedding parameters for all signals [2]. In this study, the obtained classification result by using the $k$-NN classifier was $96.3 \% \pm 1.2$. This means that the fact of considering cardiac dynamics of each subject as a different dynamic system with its own parameters, improves the quality of the extracted features for the cardiac murmur detection.

\section{Conclusions}

- The parameters associated with true state space reconstruction can be adjusted by the use of genetic algorithms joined with support vector regression for its own control parameter tuning. Additionally, the reconstruction of the diffeomorphic attractor, that is originated with all the dynamical variables, allows the estimation of features with high discriminatory capability for detecting cardiac murmurs. 
- According to the obtained results, the proposed methodology, for tuning the GA control parameters, successfully reduces its computational cost without degrading the fitness of the solution. Such a reduction is a necessary component in the proposed classification process, given the inherent computational cost of the nonlinear dynamics methods used.

- It is remarkable that the optimal embedding parameters can be found using as cost function the best possible forecast skill, in this way, good forecasting skills imply good embedding properties.

\section{Acknowledgements}

This study is framed within the project titled "Techniques of high performance computation in the automated interpretation of medical images and biosignals", financed by research direction of Manizales (DIMA) and the research vice-rectory of the National University of Colombia, code 20201004224.

\section{References}

[1] Goldberger AL, Amaral LAN, Hausdorff JM, Ivanov PC, Peng CK, Stanley HE. Fractal dynamics in physiology: Alterations with disease and aging. In Proceedings of the National Academy of science of the United States of America, volume 99. 2002; 2466-2472.

[2] Ahlstrom C, Hult P, Rask P, Karlsson JE, Nylander E, Dahlström U, Ask P. Feature extraction for systolic heart murmur classification. Annals of Biomedical Engineering 2006;34(11):1666-1677.

[3] Kantz H, Schreiber T. Nonlinear Time Series Analysis. Cambridge University Press, 2002.

[4] Takens F. Dynamical Systems and Turbulence, Lecture Notes in Mathematics, volume 898. Springer-Verlag, 1981; 366-381.

[5] Sauer T, Yorke J, Casdagli M. Embeddology. Journal of Statistical Physics 1991;65:579-616.

[6] Simon G, Verleysen M. High-dimensional delay selection for regression models with mutual information and distance-to-diagonal criteria. Neurocomputing 2007; 70:1265-1275.

[7] Hegger R, Kantz H, Olbrich E. Nonlinear analysis of physiological data. Springer, 1998; 23-47.

[8] Grassberger P, Procaccia I. Measuring the strangeness of strange attractors. Physica D 1983;9:189-208.

[9] Kennel MB, Brown R, Abarbanel HDI. Determining minimum embedding dimension using a geometrical construction. Physical Review A 1992;45:3403-3411.

[10] Cao L. Practical method for determining the minimum embedding dimension of a scalar time series. Physica D 1997; 110:43-50.

[11] Fraser AM, Swinney HL. Independent coordinates for strange attractors from mutual information. Physical Review A 1986;33:1134-1140.
[12] Babovic V, Keijzer M, Stefansson M. Chaos theory, optimal embedding and evolutionary algorithms. Technical Report 9800463, Danish Technical Research Council (STVF), 2001.

[13] Sannasiraj SA, Babovic V. Error forecasting in a wave prediction model using local linear model. In The Eighth Workshop on Ocean Models for the APEC Region (WOM8). Hong Kong, China, August 2002; .

[14] Parker TS, Chua LO. Practical Numerical Algorithms for Chaotic Systems. New York: Springer-Verlag, 1989.

[15] Tikhonov AN, Arsenin VY. Solution of Ill-Posed Problems. Washington: Winston, 1977.

[16] Whitley D. An overview of evolutionary algorithms: practical issues and common pitfalls. Information and Software Technology 2001;43(14):817-831.

[17] Grefenstette JJ. Optimization of control parameters for genetic algorithms. IEEE transactions on Systems Man and Cybernetics 1986;16(1):122-128.

[18] Wu SJ, Chow PT. Genetic algorithms for nonlinear mixed discrete-integer optimization problems via meta-genetic parameter optimization. Engeenering Optimization 1995; 24(2):137-159.

[19] Smith SF, Cicirello VA. Modeling GA performance for control parameter optimization. In Proceedings of the Genetic and Evolutionary Computation Conference. Las vegas, USA, 2000; 235-242.

[20] DeJong KA. An analysis of the Behavior of a class of Genetic Adaptative System. Ph.D. thesis, University of Michigan, 1975.

[21] Vapnik V. Statistical Learning Theory. New York: Wiley, 1998.

[22] Jaramillo J, Castellanos G. Genetic meta-algorithm based on support vector regressions. In Proceedings of the Computational Intelligence International Congress. Bogotá, Colombia, 2007; .

[23] Rosenstein M, Collins J, Luca. CD. A practical method for calculating largest lyapunov exponents from small data sets. Physica D 1993;65(1-2):117-134.

Address for correspondence:

Name: Edilson Delgado-Trejos

Full postal address: Universidad Nacional de Colombia. Campus La Nubia. Vía al aeropuerto. Oficina V-212. Manizales Caldas. Colombia. Tel: +57 3007809495 .

E-mail address: edelgadot@unal.edu.co 